\title{
Leveraging Interfaces to Improve Recommendation Diversity
}

\author{
Chun-Hua Tsai \\ University of Pittsburgh \\ 135 N Bellefield Ave \\ Pittsburgh, PA 15260 \\ cht77@pitt.edu
}

\author{
Peter Brusilovsky \\ University of Pittsburgh \\ $135 \mathrm{~N}$ Bellefield Ave \\ Pittsburgh, PA 15260 \\ peterb@pitt.edu
}

\begin{abstract}
Increasing diversity in the output of a recommender system is an active research question for solving a long-tail issue. Most of the current approaches have focused on ranked list optimization to improve recommendation diversity. However, little is known about the effect that a visual interface can have on this issue. This paper shows that a multidimensional visualization promotes diversity of social exploration in the context of an academic conference. Our study shows a significant difference in the exploration pattern between ranked list and visual interfaces. The results show that a visual interface can help the user explore a a more diverse set of recommended items.
\end{abstract}

\section{KEYWORDS}

HCI; Recommender System; Diversity; User Interface

\section{INTRODUCTION}

A recommender system is an effective approach to prevent cognitive overload when accessing information. In an era of data, users are more reliant on such systems to access on-line information. These systems can offer personalized recommendations that are based on users' preference or interests. However, personalized algorithms tend to push the user to select from a narrow set of choices. This can lead to a filter bubble effect, which shields the user from other viewpoints [5]. It can also cause an adverse effect in social fragmentation and ideological polarization of discussions on social issues [14]. Many studies have been done to prevent these negative effects by generating diversity in different disciplines, such as in online reviews [7, 30], comments [8], e-commerce [10, 23], question-answering sites [22], and politics [5, 9].

There are two main directions of work toward improving the diversity of recommendation results. The first direction, where a large portion of effort has been spent, focuses on improving the recommendation diversity of a ranked list of results. This has mainly focused on optimization by adding the "beyond relevance" factors to relevance prediction functions [2, 11, 22, 27, 32, 35]; that is, to prevent issues of over-specification by providing a recommendation list that contains diverse items. The second direction is to improve the interface of the recommender system in order to solve the

Permission to make digital or hard copies of all or part of this work for personal or classroom use is granted without fee provided that copies are not made or distributed for profit or commercial advantage and that copies bear this notice and the full citation on the first page. Copyrights for components of this work owned by others than ACM must be honored. Abstracting with credit is permitted. To copy otherwise, or republish, to post on servers or to redistribute to lists, requires prior specific permission and/or a fee. Request permissions from permissions@acm.org.

UMAP'17 Adjunct, fuly 09-12, 2017, Bratislava, Slovakia

(C) 2017 ACM. 978-1-4503-5067-9/17/07 ..\$15.00

DOI: $10.1145 / 3099023.3099073$ problem. For example, $[7,10,23,30]$ adopted the visual interface to present recommendation results with diversity aspects. The user can interact with the system beyond the ranked list. The interface represents the recommendation results with a variety of similar/dissimilar items, which can help a user to explore diverse recommendation content.

It is not surprising that all the methods for the presentation of recommendations include some kind of bias. For example, many studies pointed out the display bias in the sorted recommendation list; that is, the top-ranked items would get more user attention than lower-ranked items $[4,13]$, or the so-called position bias. More types of bias can be found in a mix or visual design recommender systems [28]. The user may have a vertical bias on a ranked list that is combined with image and search results. This finding indicates that the interface design does affect how the user interacts with the recommended items. We can say that the ranked list presentation aggravates the long-tail effect on the recommender system. However, little research effort has been focused on how the visual effect has an impact on the user interaction, as well as the distinct challenges and opportunities for designing a visual recommender system.

In this paper, we propose a user study on comparing a sorted ranked list and a two-dimensional visual interface for a social recommendation task at academic conferences. The experiment result indicates a significant difference in the exploration patterns of these interfaces. We found that the user visual interface group explored more diverse social connections from the system. In the subjective evaluation, however, the ranked list was evaluated higher in user's intention to reuse the system. The main contribution of this paper is to suggest and evaluate an interface to solve the "longtail" issue within the recommender system. The findings support the overall concept of interface design helping the user to fairly explore the recommender system results. We argue that a user can intuitively access diverse recommendations if we provide that user with an accurate perception of how the data is presented. This finding provides some insights towards the development of a diversity-supporting interface to access recommendations online.

\section{RELATED WORK}

\subsection{Recommendation Diversity}

The recommender system aims to facilitate the presentation of useful information and reduce information overload. As a result, an efficient system is required to deliver the relevant items with a high degree of accuracy, based on user preferences or behavior [24]. Many scholars have criticized highly accurate recommender algorithms that provide the user with a narrow set of choices or 
limited information exposure [5], which is a well-known overspecification problem in the domain of recommender systems [1, 33]. Some scholars argued for the negative filter bubble effect if the user can only access to a "biased" recommendation result based on personalized social network or ideological preferences [9]. It might be necessary to diversify the recommendation results to prevent this bias. However, the definition of diversity varies and lacks a universal metric and scale. Diverse items may be generated based on user personalities for wider exposure [32]. For example, in online news consumption, it may be possible to show differing opinions for the purposes of comparison [14]. Some studies tried to provide a balanced choice between novel and similar items in a recommender ranked list [11,27, 29], or to provide categorical coverage that was as broad as possible [20, 23, 31]. A recommender system that is specifically designed to facilitate the diversity of results needs to carefully consider the differences between the provided results.

\subsection{Improving Diversity}

To address the diversity problem, much effort has been spent on increasing the diversity of ranked list results offered to the users of recommender systems. The work can be separated into three categories: 1) Learning to rank: an approach to aggregate the novelty and accuracy in a ranked list. For instance, a hybrid algorithm can extend the recommended list with the diversity, novelty, and accuracy of items [2, 11, 15, 22, 27, 32, 35]. 2) Content-based diversification: to provide diverse content based on the varying similarity metrics, e.g. opinion similarities [8], aspect similarities [29], and topic similarities [33]. 3) Collaborative filtering-based diversification: to extend the collaborative network by probabilistic approaches [1, 36] and latent distance [17]. However, all of these approaches have focused on the diversification within the ranked list. The ensembled ranked list lacked perception of the properties behind the recommendation result. Users often lower their satisfaction with or trust in the system if it has provided a list that conflicts with their expected levels of similarity [16].

Providing a visual interface is another approach to solving the diversity recommendation problem. Some examples of a visual discovery interface include the CTR rate in an e-commerce website [23]; the two-column format to present a two-sided opinion on controversial subjects [14]; and the distance of latency among users' ideology [9] through an explainable interface to justify the reason for providing recommendations for a user to actively access diverse content [33,34]. [19] designed an interface to provide an explanation to justify the recommendation results, which has been shown to be useful for the user to understand the reasoning behind particular recommendation results. The user can choose to explore the system further, based on the explanation. [21] proposed a user-controllable interface that would allow the user to change each featurefis weighting to provide a more personalized ranking. [7, 10, 30] all proposed various interfaces that show diverse recommendation results. However, these studies have focused on individual metrics, such as satisfaction and helpfulness, among others. A study from an objective perspective has not yet been performed, and it remains unknown as to which interface design can truly help users consume more diverse results.

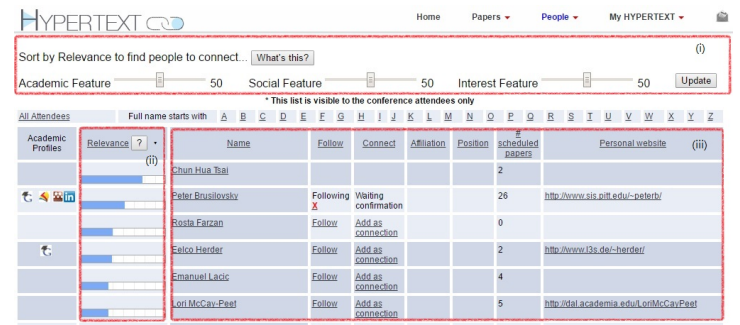

Figure 1: A screenshot of the RelExplorer system [26]: (i) A control panel of three feature sliders. (ii) A ranked list of the personalized relevance scores. (iii) The user profile information from the Conference Navigator system [6].

\subsection{Perceiving Diversity}

Humans are naturally connected to or prefer to interact with people who hold similar beliefs - the homophily effect [9]. The social recommender system should provide more diverse content for a user to extend their social connections outside of a personal bubble. However, not every user equally values the diversity of provided recommendations [3]. The desired level of diversity differs from individual to individual. For instance, [16] classified people into two categories: "diversity seeking" and "challenge averse" to describe the relationship between stratification and level of diversity exposure. This classification may explain the individual differences in the information-seeking process. The social recommender system with included diversity needs a different interface to fit the needs of users' prior convictions. Furthermore, simply presenting conflicting information may not help users to interact with diverse content. A reinforcing effect may happen if the user feels threatened by unfamiliar information [14]. The study of [30] adopted dimension-reduction techniques to project the data in two or three dimensions for visualization purposes. However, the user was not able to distinguish the meaning of each axis, which led the user to explore opinions that were similar to their own [7]. [5] argues that the "diverse conceptions of democracy" must be considered when designing a diversity-improving tool. It is necessary to realize the visual effect before we can design a general interface for enhancing recommendation diversity.

\section{APPROACH}

\subsection{The Context}

The work presented in this paper has been performed in the context of developing a social recommendation function for the Conference Navigator system [6]. This function was added to the original system to help conference attendees to connect with other scholars at the venue. The overall goal of the social recommendation function was to help conference attendees explore potential social links while attending events. Our recommendation approach was designed to emphasize different aspects of similarity that could be valuable to identify people of interest. These aspects were encapsulated in three separate recommender engines that accounted for the similarity between publications (academic), co-authorship similarities (social), and the similarity of interest in conference papers, expressed as bookmarks (interest). We first designed a standard 


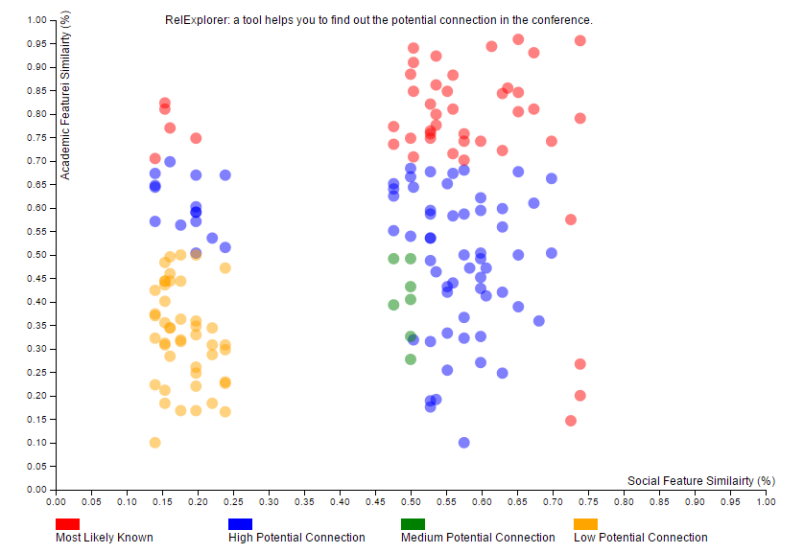

Figure 2: A two-dimensional scatter plot to combine the recommendation results of two engines. Each axis represents a recommender engine (a feature), each node represents an attendee at the conference, and its location is determined by its feature weights (here - the Academic feature for $\mathrm{Y}$ and the Social feature for $\mathrm{X}$ ). A click on a node opens a new window to explore profile details.

recommendation interface with a ranked list and a control panel to change the weighting of contributing engines. This version was explored in the pilot study. The results of the pilot study briefly presented in the next subsection helped us to design a more advanced visual interface, which is presented below.

\subsection{The Pilot Study}

In our pilot study, we explored to what extent users were able to explore a diverse space of conference attendees by using a controlled fusion of several focused recommender engines, an approach that we successfully used in the past [18]. Figure 1 presents a screenshot of the pilot study interface. The interface allowed users to fuse three recommendation components into a single. To control the fusion, the user could change the weighting of each component based on individual preference. For example, the user may increase the Academic feature to find more conference attendees with similar research interests, or increase the Social feature to find conference attendees with a similar co-authorship network.

The results of the pilot study were presented in [26]. In brief, we found that users did use the control panel to explore social connections. However, the exploration pattern showed that only a small set of the recommended items had been explored. That is, most of the exploration focused on the group of people with a high relevance score. The group with a low relevance score was less likely to be explored. However, the lower relevance score and rank may be due to a lack of user profile data (such as junior scholars who have fewer publications) or the presentation bias of the ranked list interface. It does not determine the real social value to users. The pilot study showed the limitations of the ranked-list interface, even in the presence of user control. This work motivated the current study presented below: reducing the presentation through a visual interface.

\subsection{Visual Interface}

A ranked list can only represent recommended items in a single dimension. It forces the system to use the ensembled or controlled fusing method to mix multiple aspects of relevance into a single list. However, the pilot study showed that the ranked list caused an exploration pattern with little diversity. To avoid ranking bias, we explored a two-dimensional interface design that independently presents two recommendation aspects. We found that a scatter plot is a universal design to represent multidimensional data [12]. Hence, we developed a scatter plot interface to show the recommended results in two dimensions (Figure 2).

A scatter plot diagram allows us to simultaneously present more than one recommendation feature. For example, we can display the Academic feature on the $\mathrm{X}$ axis and the Social feature on the $\mathrm{Y}$ axis. The two features define the position of each recommended item in the scatter plot. The interface can help users intuitively perceive the relative relationship between the two recommendation features and can help them quickly focus on a scatter plot area with a specific combination of two recommendation features (for example, the upper left corner with high academic and low social similarity), instead of changing the feature weighting in the ranked list. To focus user attention on areas with meaningful combinations of features, we used color coding to represent four groups of recommended items: high relevance in both features $(\mathrm{HH})$, low relevance in both features (LL), and high relevance in one or the other of the features (HL or LH). We realized the users may find it difficult to understand the meaning of these four groups, so we set the titles based on the prospective effect of each cluster; i.e. the red color of the HH group indicates the conference attendees who may be most likely known, due to their high academic and social similarity to the user. The yellow color of the LL group is the group of attendees who may have a low potential connection, due to the low values of both of the features. The blue and green colors show the attendees with only one high similarity feature, which are assigned as high potential connections and medium potential connections.

Since the features represented in the visualization were computed by two different recommender engines, the features were not comparable without normalization. We adopted a standard Z-Score to normalize all the features to the same scale, from 0 to 1 . The function was defined as: ZScore $=\frac{x_{i}-u_{j}}{\sigma_{j}}$, where $x_{i}$ is $i_{t h}$ recommended item and $j$ represents the corresponding two features. Then, we used a standard Z-table to convert the ZScore to the corresponding percentile $p_{i j}$. This allowed us to list all the features on the same scale for both the ranked list and scatter plot diagram.

\section{EXPERIMENT}

\subsection{Data and Participants}

We used the Conference Navigator system with the extended social recommendation function [26] for assessing and comparing the two proposed interfaces. The pilot study of the ranked list interface was conducted at the HT 2016 and the UMAP 2016 conferences in Halifax, Nova Scotia, Canada. The study of the visual interface was performed at the CIC 2016 conference in Pittsburgh, Pennsylvania, USA. The conference publication and attendee lists were used to compute recommendations based on three types of similarity (the features mentioned in Section 3.1). For Study 1, we recruited 12 
participants (nine male and three female) from the HT\&UMAP 2016 conferences. For Study 2, we recruited 14 participants (seven male and seven female) from the CIC 2016 conference. All of the participants were registered conference attendees with at least one publication paper in the system. The age of the participants ranged from 20 to 40 years old, and they were recruited at the conference. All of them were graduate students or at the junior research level at their associated academic institution or industry research lab.

\subsection{Experiment Design and Procedure}

To evaluate the effect of the proposed interface on the exploration of social recommendations by academic attendees, we designed a user study that combined both a subjective and an objective evaluation. The ranked list interface (Group 1) was used as the control group, and the scatter plot interface (Group 2) was the experimental group. We recorded the system activity log while each participant used the system. Both studies used the prepared laptop and were performed under the control of the first author directly at the conference venue.

We asked participants to complete two tasks using the designated system and to fill out a post-task questionnaire. The tasks were based on different social needs that would arise while using a social recommendation system. In each task, participants were asked to follow two people, according to the instructions. Task1: Find two conference attendees you already know. a) Decide whether you need to follow them or connect to them in the system. b) Examine information about these attendees to find out how these two people can help to establish new connections at this conference. Task2: Explore two conference attendees you a) donfit know in person yet and b) you are interested in meeting. Examine information about these attendees to find out who could introduce you to them or how you could introduce yourself.

In order to examine to what extent each interface encouraged diversity in exploring conference attendees, we observed the user interaction with four different "quadrants" that represented combinations of two features [25], e.g. high academic and high social features, high academic and low social features, and so on. In order to see how the participants interacted with different groups, we defined two kind of measurements - Diversity and Coverage. Both of the metrics were measured by Entropy: $d_{u}=-\sum_{i=1}^{4} p_{i} \log _{4} p_{i}$, where $p_{i}$ was the probability for a particular quadrant and the proportion of all the userfis selection (following) [15]. The metric of Coverage was similar in diversity but omitted the quantity in each quadrant to see how usersfi exploration covered all four quadrants of the recommended items. The Coverage metric was only compared between groups, due to each task only having two follow-up activities. The action of clicking and the amount of time spent were also recorded in this experiment.

\section{RESULTS}

\subsection{Data Analysis}

A non-parametric Wilcoxon signed-rank test of difference among the metrics was conducted and rendered a $\mathrm{W}$ value that indicated a significant difference when $p<0.05$. The results are reported in Tables 1 and 2. They indicate that the diversity (entropy) and coverage metrics were significantly different among groups. This result

\begin{tabular}{|l|c|c|c|c|}
\hline Metric & Group 1 (Ave.) & Group 2 (Ave.) & $\mathrm{W}$ & P-value \\
\hline Diversity & 0.14 & 0.26 & 80 & $8 \mathrm{e}-06\left(^{*}\right)$ \\
\hline Coverage & 0.29 & 0.53 & 30 & $0.005\left(^{*}\right)$ \\
\hline Click & 2.58 & 4.11 & 300 & 0.2 \\
\hline Time & 222 & 173 & 300 & 0.8 \\
\hline
\end{tabular}

Table 1: The differences between the Group 1: Ranked List and Group 2: Scatter plot. The Diversity and Coverage metric reflects how the participants "followed" the conference attendees. $\left({ }^{*}\right)$ means significant differences at the $5 \%$ level (p-value $<0.05$ )

\begin{tabular}{|l|c|c|l|c|}
\hline Metric & Task 1 (Ave.) & Task 2 (Ave.) & W & P-value \\
\hline Diversity & 0.20 & 0.21 & 300 & 0.8 \\
\hline Click & 2.24 & 4.52 & 200 & $0.002\left(^{*}\right)$ \\
\hline Time & 165 & 228 & 300 & 0.7 \\
\hline
\end{tabular}

Table 2: The difference between the Task 1: Explore known connections and Task 2: Explore not known connections

supports the concept that the scatter plot interface led the user to explore a more diverse set of attendees across the four quadrants. Our findings demonstrate the potential for showing recommendations in a multidimensional view to help or change the diversity of the exploration pattern. We also found that the click count was significantly different between tasks: it required significantly more work (measured by clicks) to find new connections. The data also show that users in the scatter plot group needed more clicks to complete their tasks (most likely because the scatter plot did not immediately show the participants' names, which did appear in the ranked list). At the same time, the users of the scatter plot interface spent less time to complete the tasks. While these differences are not statistically significant, it could indicate that the scatter plot interface helped users to quickly spot social links of interest.

\subsection{Exploration Patterns}

Figure 3 shows the exploration parameters for the two proposed tasks performed with the interfaces. We can see a clear difference between the two interfaces. The participants who used the ranked list interface were more focused on the high-relevance group; i.e. items with high similarity for both the academic and social features. The recommended items (conference attendees) with low similarity in both recommendation features were less explored and less followed by the participants. In contrast, the participants who were using the scatter plot interface showed a more diverse exploration pattern - they more extensively examined items with both high and low similarities in each dimension. This result supports the expectation that the proposed scatter plot interface helped the participants to explore attendees who fell outside of the ranking bias area, i.e., items that were top ranked with a high degree of similarity.

\subsection{Post-Questionnaire Analysis}

Figure 4 shows the post-questionnaire analysis. We found that both of the interfaces had a high score on subjective feedback. For example, both of the interfaces were found to be informative and 
(a) Exploration Pattern on Ranked List Interface

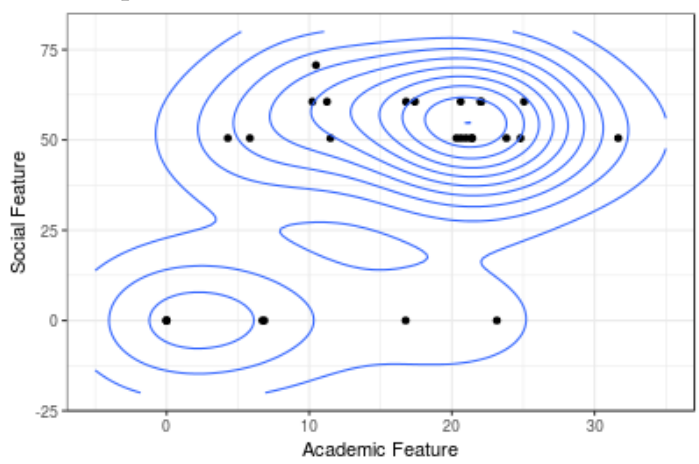

(c) Following Pattern on Ranked List Interface

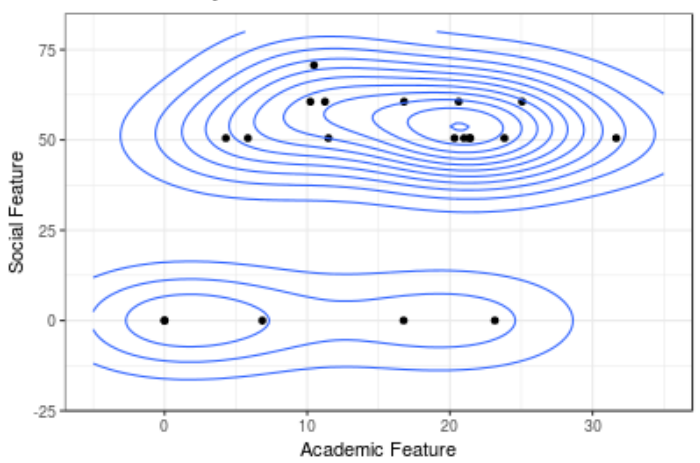

(b) Exploration Pattern on Scatter plot Interface

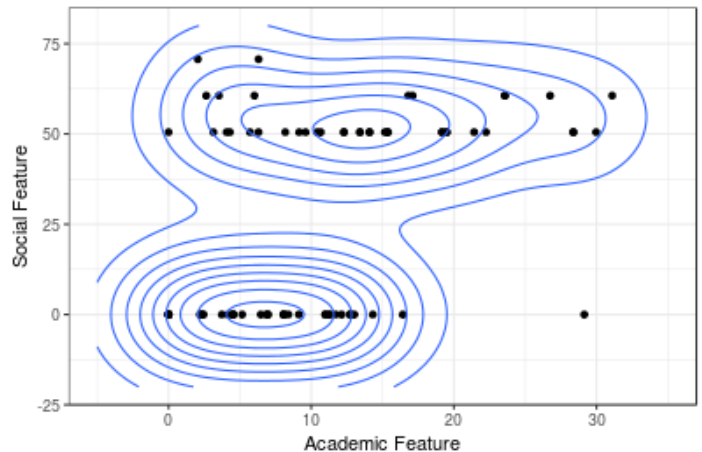

(d) Following Pattern on Scatter plot Interface

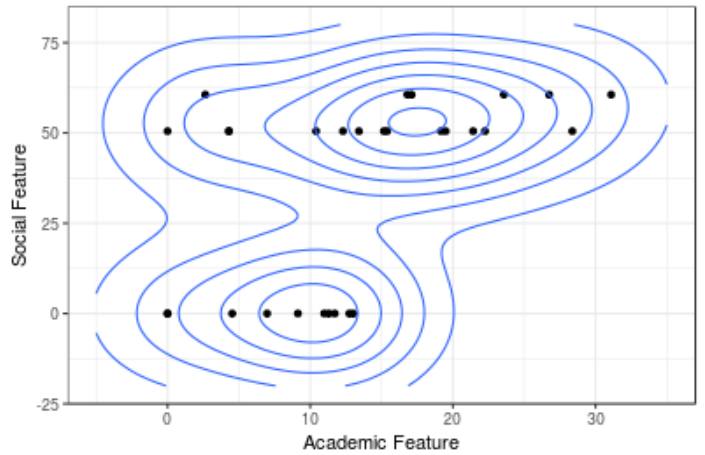

Figure 3: The social exploration patterns of the user study. Both of the exploration and following activities are recorded from the "explore unknown attendees task".

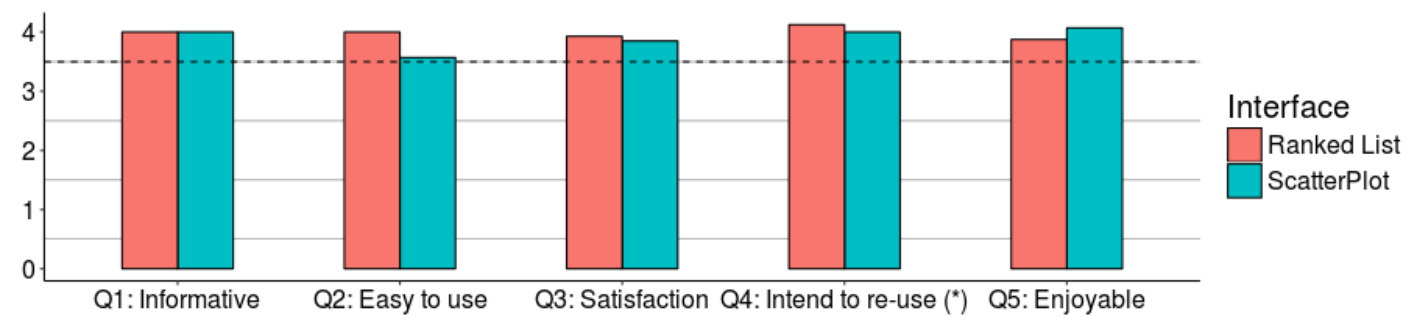

Figure 4: Usability and user satisfaction data. $\left(^{*}\right)$ means a significant difference at the $5 \%$ level (p-value $\left.<0.05\right)$

easy to use. On average, the ranked list scored higher on ease of use, satisfaction, and intention to re-use, while the visual "scatter plot" interface scored higher on enjoyment. However, only intention to re-use was significantly different between the scatter plot interface and the ranked list interface. This may be due to the fact that the scatter plot decreased availability of detailed information such as name, affiliation, and relevance score (shown in Figure 1). This could also explain why it was necessary for a user to perform more clicks to interact with recommendation items of interest. This finding confirms the diverse exploration patterns on the visual interface, but it also presents the challenge of creating such an interface while maintaining the usability level of the baseline interface.

\section{CONCLUSION}

In this paper, we proposed a new visual interface to display social recommendation results produced by several recommender engines. We showed that the visual interface encourages users to explore a more diverse set of recommended items. We also showed that the exploration patterns were considerably different between the visual and the baseline interfaces. The analysis hinted that the new visual interface required less time, but more interaction steps to complete the same tasks, as compared to the baseline interface. The new visual interface had a lower score on the intention to reuse as evaluated by the user feedback. The results indicated that visual interface can help users to improve exploration diversity, but 
that further design work would be required to match the level of usability of the baseline system.

In the current mainstream recommender system, more attention is paid to the problem of presentation optimization [28]; that is, to optimize the layout in a web page with several recommendation functions. We believe that this issue requires a greater level of understanding on how visual effects can influence users interactions with a recommender system. The interface of a recommender system could facilitate different exploration goals. In this study, we showed that a diversity-oriented interface can help the user to explore a more diverse set of recommended items. It sheds light on the issue of how to design an interface for a diversity exploration task, but adds a challenge to maintain the systemfis overall usability.

We plan to continue our work on recommender system interfaces that help users further explore diverse recommendations. New designs will maintain the recommendation model accuracy and user satisfaction at the same levels or will show the trade-off between the metrics. The present study does have some limitations. First, we explored a combination of just two recommendation features. The interface will need more work to explore more than two features. Second, the scatter plot interface made it difficult to recognize familiar names. The user needed to click on many dots to fully inspect the recommended results. To address this issue, we plan to explore a combination of the scatter plot and ranked list interfaces.

\section{REFERENCES}

[1] Panagiotis Adamopoulos and Alexander Tuzhilin. 2014. On over-specialization and concentration bias of recommendations: Probabilistic neighborhood selec tion in collaborative filtering systems. In Proceedings of the 8th ACM Conference on Recommender systems. ACM, 153-160.

[2] Gediminas Adomavicius and YoungOk Kwon. 2012. Improving aggregate recommendation diversity using ranking-based techniques. IEEE Transactions on Knowledge and Data Engineering 24, 5 (2012), 896-911.

[3] Jisun An, Daniele Quercia, and Jon Crowcroft. 2013. Why individuals seek diverse opinions (or why they don't). In Proceedings of the 5th Annual ACM Web Science Conference. ACM, 15-18.

[4] Judit Bar-Ilan, Kevin Keenoy, Mark Levene, and Eti Yaari. 2009. Presentation bias is significant in determining user preference for search resultsfi!?A user study. fournal of the Association for Information Science and Technology 60, 1 (2009) 135-149.

[5] Engin Bozdag and Jeroen van den Hoven. 2015. Breaking the filter bubble democracy and design. Ethics and Information Technology 17, 4 (2015), 249-265.

[6] Peter Brusilovsky, Jung Sun Oh, Claudia López, Denis Parra, and Wei Jeng. 2016. Linking information and people in a social system for academic conferences. New Review of Hypermedia and Multimedia (2016), 1-31.

[7] Siamak Faridani, Ephrat Bitton, Kimiko Ryokai, and Ken Goldberg. 2010. Opinion space: a scalable tool for browsing online comments. In Proceedings of the SIGCHI Conference on Human Factors in Computing Systems. ACM, 1175-1184.

[8] Giorgos Giannopoulos, Marios Koniaris, Ingmar Weber, Alejandro Jaimes, and Timos Sellis. 2015. Algorithms and criteria for diversification of news article comments. Fournal of Intelligent Information Systems 44, 1 (2015), 1-47.

[9] Eduardo Graells-Garrido, Mounia Lalmas, and Ricardo Baeza-Yates. 2016. Data Portraits and Intermediary Topics: Encouraging Exploration of Politically Diverse Profiles. In Proceedings of the 21st International Conference on Intelligent User Interfaces. ACM, 228-240.

[10] Rong Hu and Pearl Pu. 2011. Helping Users Perceive Recommendation Diversity. In DiveRS@RecSys.43-50.

[11] Neil Hurley and Mi Zhang. 2011. Novelty and diversity in top-n recommendationanalysis and evaluation. ACM Transactions on Internet Technology (TOIT) 10, 4 (2011), 14.

[12] Hannah Kim, Jaegul Choo, Haesun Park, and Alex Endert. 2016. Interaxis: Steering scatterplot axes via observation-level interaction. IEEE transactions on visualization and computer graphics 22, 1 (2016), 131-140.

[13] Kristina Lerman and Tad Hogg. 2014. Leveraging position bias to improve peer recommendation. PloS one 9, 6 (2014), e98914.

[14] Q Vera Liao and Wai-Tat Fu. 2013. Beyond the filter bubble: interactive effects of perceived threat and topic involvement on selective exposure to information.
In Proceedings of the SIGCHI conference on human factors in computing systems. ACM, 2359-2368.

[15] Jennifer Moody and David H Glass. 2016. A Novel Classification Framework for Evaluating Individual and Aggregate Diversity in Top-N Recommendations. ACM Transactions on Intelligent Systems and Technology (TIST) 7, 3 (2016), 42.

[16] Sean A Munson and Paul Resnick. 2010. Presenting diverse political opinions: how and how much. In Proceedings of the SIGCHI conference on human factors in computing systems. ACM, 1457-1466.

[17] Tien T Nguyen, Pik-Mai Hui, F Maxwell Harper, Loren Terveen, and Joseph A Konstan. 2014. Exploring the filter bubble: the effect of using recommender systems on content diversity. In Proceedings of the 23rd international conference on World wide web. ACM, 677-686.

[18] Denis Parra and Peter Brusilovsky. 2015. User-controllable personalization: A case study with SetFusion. International fournal of Human-Computer Studies 78 (2015), 43fi?!67.

[19] Pearl Pu and Li Chen. 2007. Trust-inspiring explanation interfaces for recommender systems. Knowledge-Based Systems 20, 6 (2007), 542-556.

[20] Shameem A Puthiya Parambath, Nicolas Usunier, and Yves Grandvalet. 2016. A coverage-based approach to recommendation diversity on similarity graph. In Proceedings of the 10th ACM Conference on Recommender Systems. ACM, 15-22.

[21] J Ben Schafer, Joseph A Konstan, and John Riedl. 2002. Meta-recommendation systems: user-controlled integration of diverse recommendations. In Proceedings of the eleventh international conference on Information and knowledge management. ACM, 43-51.

[22] Idan Szpektor, Yoelle Maarek, and Dan Pelleg. 2013. When relevance is not enough: promoting diversity and freshness in personalized question recommendation. In Proceedings of the 22nd international conference on World Wide Web. ACM, 1249-1260.

[23] Choon Hui Teo, Houssam Nassif, Daniel Hill, Sriram Srinivasan, Mitchell Goodman, Vijai Mohan, and SVN Vishwanathan. 2016. Adaptive, Personalized Diversity for Visual Discovery. In Proceedings of the 10th ACM Conference on Recommender Systems. ACM, 35-38.

[24] Chun-Hua Tsai. 2016. A Fuzzy-Based Personalized Recommender System for Local Businesses. In Proceedings of the 27th ACM Conference on Hypertext and Social Media. ACM, 297-302.

[25] Chun-Hua Tsai. 2017. An Interactive and Interpretable Interface for Diversity in Recommender Systems. In Proceedings of the 22Nd International Conference on Intelligent User Interfaces Companion (IUI '17 Companion). ACM, New York, NY, USA, 225-228. DOI : http://dx.doi.org/10.1145/3030024.3038292

[26] Chun-Hua Tsai and Peter Brusilovsky. 2017. Providing Control and Transparency in a Social Recommender System for Academic Conferences. In Proceedings of the 2017 Conference on User Modeling Adaptation and Personalization. ACM.

[27] Saúl Vargas and Pablo Castells. 2011. Rank and relevance in novelty and diversity metrics for recommender systems. In Proceedings of the fifth ACM conference on Recommender systems. ACM, 109-116.

[28] Yue Wang, Dawei Yin, Luo Jie, Pengyuan Wang, Makoto Yamada, Yi Chang, and Qiaozhu Mei. 2016. Beyond ranking: Optimizing whole-page presentation. In Proceedings of the Ninth ACM International Conference on Web Search and Data Mining. ACM, 103-112.

[29] Jacek Wasilewski and Neil Hurley. 2016. Intent-Aware Diversification Using a Constrained PLSA. In Proceedings of the 10th ACM Conference on Recommender Systems. ACM, 39-42.

[30] David Wong, Siamak Faridani, Ephrat Bitton, Björn Hartmann, and Ken Goldberg. 2011. The diversity donut: enabling participant control over the diversity of recommended responses. In CHI'11 Extended Abstracts on Human Factors in Computing Systems. ACM, 1471-1476.

[31] Le Wu, Qi Liu, Enhong Chen, Nicholas Jing Yuan, Guangming Guo, and Xing Xie. 2016. Relevance meets coverage: A unified framework to generate diversified recommendations. ACM Transactions on Intelligent Systems and Technology (TIST) 7, 3 (2016), 39

[32] Wen Wu, Li Chen, and Liang He. 2013. Using personality to adjust diversity in recommender systems. In Proceedings of the 24th ACM Conference on Hypertext and Social Media. ACM, 225-229.

[33] Cong Yu, Laks Lakshmanan, and Sihem Amer-Yahia. 2009. It takes variety to make a world: diversification in recommender systems. In Proceedings of the 12th international conference on extending database technology: Advances in database technology. ACM, 368-378.

[34] Cong Yu, Laks VS Lakshmanan, and Sihem Amer-Yahia. 2009. Recommendation diversification using explanations. In Data Engineering, 2009. ICDE'09. IEEE 25th International Conference on. IEEE, 1299-1302.

[35] Tao Zhou, Zoltán Kuscsik, Jian-Guo Liu, Matúš Medo, Joseph Rushton Wakeling, and Yi-Cheng Zhang. 2010. Solving the apparent diversity-accuracy dilemma of recommender systems. Proceedings of the National Academy of Sciences 107, 10 (2010), 4511-4515.

[36] Xiaojin Zhu, Andrew B Goldberg, Jurgen Van Gael, and David Andrzejewski. 2007. Improving Diversity in Ranking using Absorbing Random Walks.. In HLT-NAACL. 97-104. 